\section{Fatores associados à ocorrência de cesárea e aborto em mulheres selecionadas em um centro de saúde no município do Rio de Janeiro, Brasil}

\section{Factors associated to cesarean sections and abortions in women selected from a health clinic in the city of Rio de Janeiro, Brazil}

\author{
Gilberto Kac 1 \\ Erika Aparecida Silveira 2 \\ Lívia Costa de Oliveira 3 \\ Daniele Marano Rocha Araújo 4 \\ Elton Bicalho de Sousa 5
}

\begin{abstract}
Objectives: to investigate factors potentially associated to cesarean sections and abortions.

Methods: data from a cohort study during postpartum were analyzed for 352 women aged 15-45 years old. The following outcomes were studied: cesarean sections and abortions prior to current pregnancy. Statistical analysis included multivariate logistic regression models with hierarchical approach.

Results: c-sections and abortions prevalence were $36.3 \%$ and $34.0 \%$, respectively. The final model indicated that the following variables remained associated to cesarean sections: level 1: Caucasian white $(O R=2.02 ; 95 \% C I: 1.29-3.16)$; level 2: tubal ligation $(O R=19.68 ; 95 \% C I: 5.77-67.15)$. The following $v a-$ riables remained associated to abortion occurrence: level 1: age $\geq 29$ years $(O R=6.11 ; 95 \% C I$ : 2.9412.72); marital status: married $(O R=4.22 ; 95 \% C I$ : 2.03-8.78), single (OR=3.70; 95\%CI: 1.59-8.61).

Conclusions: white Caucasian women and tubal ligation were potentially associated co-variables for cesarean sections performance while marital status and maternal age were associated to abortions, prevalent in women over 29 years old.
\end{abstract}

Key words Cesarean section, Abortion, Race, Marital status, Body mass index

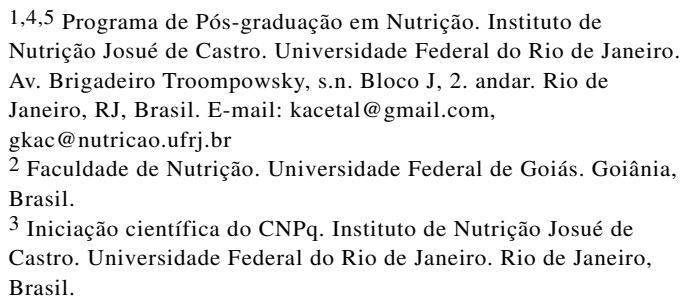
Nutrição Josué de Castro. Universidade Federal do Rio de Janeiro. Av. Brigadeiro Troompowsky, s.n. Bloco J, 2. andar. Rio de Janeiro, RJ, Brasil. E-mail: kacetal@gmail.com, gkac@nutricao.ufrj.br

2 Faculdade de Nutrição. Universidade Federal de Goiás. Goiânia, Brasil.

3 Iniciação científica do CNPq. Instituto de Nutrição Josué de Castro. Universidade Federal do Rio de Janeiro. Rio de Janeiro, Brasil.

\section{Resumo}

Objetivos: investigar fatores potencialmente associados à ocorrência de cesárea e aborto.

Métodos: foram analisados dados de uma coorte no pós-parto com 352 mulheres entre 15-45 anos. Os seguintes desfechos foram estudados: ocorrência de cesárea no último parto e ocorrência de aborto anterior à última gravidez. A análise estatística foi feita por meio de modelos de regressão logística multivariados e hierarquizados.

Resultados: as prevalências de cesárea e aborto foram de $36,3 \%$ e 34,0\%, respectivamente. O modelo final revelou que as seguintes variáveis permaneceram estatisticamente associadas à ocorrência de cesárea: nível 1: cor de pele branca $(O R=2,02$; IC95\%: 1,29-3,16); nível 2: ligadura $(O R=19,68$; IC95\%: 5,77-67,15). As seguintes variáveis permaneceram associadas à ocorrência de aborto: nível 1: idade $\geq 29$ anos (OR=6,11; IC95\%: 2,94-12,72), estado marital: vive em união $(O R=4,22 ;$ IC $95 \%$ : 2,03-8,78); solteira: (OR=3,70; IC95\%: 1,59-8,61).

Conclusões: a cor de pele branca e a prática de ligadura foram co-variáveis potencialmente associadas à ocorrência de cesárea, enquanto o estado marital em união ou solteira e a idade materna estiveram associadas à ocorrência de aborto, sendo maior a probabilidade para mulheres acima de 29 anos.

Palavras-chave Cesárea, Aborto, Raça, Estado civil, Índice de massa corporal 


\section{Introdução}

O bom prognóstico da gestação é de grande importância para saúde materno-infantil. Neste contexto é fundamental que sejam acumuladas mais informações sobre os fatores associados a cesárea e ao aborto.

Tem-se observado nos últimos 25 anos uma epidemia de cesarianas. Em relação ao panorama mundial, a taxa de cesárea na Coréia do Sul, em 2001 , foi de $36,6 \%$, na Itália foi de 33,2\% em 2000, 1 no México foi de 33,3\% em $1985^{2}$ e no Brasil foi de $27,1 \%$ em 1998. 3 Os Estados Unidos, no ano de 2004 , atingiram a taxa de $29,1 \%$, sendo esta considerada a mais alta já relatada naquele país. $1,3,4$

Recentemente o Brasil conseguiu deixar o incômodo posto de campeão de cesarianas, hoje ocupado pelo Chile. Esse fato pode ser parcialmente creditado a uma medida adotada pelo Ministério da Saúde, que fixou limite de $40 \%$ nas cesarianas feitas pelo Sistema Único de Saúde (SUS), deixando de pagar os custos com esse procedimento quando o limite é excedido. Dados mais recentes no Brasil revelam que a taxa foi de $39,7 \%$ em 2002.5 A incidência de cesáreas no país ainda varia segundo o tipo de hospital; 24,3\% em hospital escola e $89,2 \%$ em hospital privado, como observado por Fabri et al.,6 e também com estado nutricional pré-gestacional; $53,3 \%$ em mulheres obesas e $24,5 \%$ em mulheres com baixo peso. 7

Existem inúmeros fatores associados com a ocorrência de cesárea. Os principais incluem a idade materna, a cor de pele, o nível socioeconômico, o estado nutricional pré-gestacional, ganho de peso gestacional, a paridade e a ocorrência prévia de cesárea. 6,8

Em relação à ocorrência de aborto, sabe-se que se trata de um outro desfecho de interesse para a saúde pública.9,10 Desde o fim dos anos 80 e início dos anos 90 vem sendo observado um aumento no número de abortos em hospitais da rede pública, sendo que nos últimos dez anos esse aumento tem sido ainda mais expressivo.10 Em 1990 o aborto figurava como a quarta causa de mortalidade materna no país, e Schor ${ }^{9}$ constatou que em cinco anos tinham duplicado, proporcionalmente, o número de abortos em relação ao número de partos. Dados recentes de um estudo 11 de base populacional com 3002 mulheres entre 15 e 49 anos, realizado em Pelotas, Rio Grande do Sul, revelaram que o aborto foi provocado por $7 \%$ da amostra, variando de $1,6 \%$ a $9 \%$ entre mulheres de 15 a 19 anos e 40 a 49 anos, respectivamente. Por outro lado, os resultados de Menezes et al. 12 revelaram, a partir de um estudo transversal de base domiciliar, realizado no Rio de Janeiro, Salvador e Porto Alegre, frequiências de aborto mais elevadas. Esses autores observaram uma frequência mais elevada de aborto entre as mulheres com baixa escolaridade e as que possuíam parceiros ocasionais.

Alguns estudos associam o aborto principalmente com o consumo de álcool e fumo. 13,14 Entre os fatores determinantes, devemos destacar ainda o efeito da idade, do estado marital e o do estado nutricional pré-gestacional, embora o efeito deste último ainda não esteja claramente evidenciado pela literatura. ${ }^{11}$ Níveis deficientes de micronutrientes como o ácido fólico, ferro e zinco também são citados na literatura como potenciais fatores abortivos. ${ }^{15-17}$

Dentro desse contexto, o objetivo do presente estudo foi investigar o efeito de fatores sóciodemográficos, da saúde reprodutiva pregressa e do estado nutricional pré-gestacional, associados à ocorrência de cesárea e aborto em mulheres acompanhadas durante nove meses pós-parto, no muncípio do Rio de Janeiro.

\section{Métodos}

Quatrocentas e setenta e nove mulheres foram recrutadas e acompanhadas por nove meses pós-parto, em um estudo de seguimento, realizado no Município do Rio de Janeiro, entre maio de 1999 e abril de 2001. Informações complementares sobre o projeto original podem ser obtidas em outras publicações. 18-21 Como os dados referentes aos desfechos reprodutivos (cesárea e aborto) só foram investigados aos seis meses pós-parto, foram incluídas na presente análise apenas as 352 mulheres que alcançaram a terceira onda de seguimento.

O estudo original envolveu quatro ondas de seguimento: aos 15 dias, dois, seis e nove meses aproximadamente, período em que os dados antropométricos e todas as co-variáveis foram coletadas. As mulheres foram recrutadas em dois locais distintos, a saber: na maternidade central da área Oswaldo Nazareth - Praça XV, durante a rotina de pré-natal, e durante a rotina de imunização do BCG no próprio Centro Municipal de Saúde Marcolino Candau. Cabe ressaltar que a captação na rotina prénatal consistia na seleção de gestantes com mais de sete meses e posterior agendamento ao nascimento do bebê. Assim, ao nascer o bebê, a mãe deveria procurar a equipe do projeto com aproximadamente 15 dias pós-parto.

Os critérios de elegibilidade estabelecidos para entrada na coorte foram: ter entre 15 e 45 anos, ter 
menos de 30 dias de pós-parto na data da primeira entrevista, estar livre de doenças crônicas, apresentar idade gestacional $\geq 35$ semanas ao nascimento, não apresentar gestação gemelar e residir na área programática do estudo.

O padrão de perdas foi avaliado levando em consideração a distribuição da taxa final de seguimento (número de mulheres com seguimento completo/número de mulheres que entraram na coorte), segundo diversas co-variáveis importantes (faixa etária, renda em quartis, estado marital e escolaridade, entre outras). Diferenças nas taxas finais de seguimento foram avaliadas segundo o teste do quiquadrado para proporções.

O projeto original foi submetido e aprovado pela Comissão de Ética do Núcleo de Estudos de Saúde Coletiva (NESC), da Universidade Federal do Rio de Janeiro (UFRJ), e está de acordo com a Resolução 196/96, do Conselho Nacional de Saúde.22 Todas as participantes assinaram termo de consentimento, que foi obtido de forma livre e espontânea, após terem sido feitos todos os esclarecimentos necessários.

Os desfechos reprodutivos, isto é, variáveis dependentes anlisadas, foram ocorrência de cesárea no último parto (sim, não) e de aborto em qualquer gravidez anterior (sim, não). Esses desfechos foram informados pela mulher no momento do terceiro acompanhamento, aos seis meses pós-parto.

Foram construídos modelos teóricos de determinação dos desfechos reprodutivos especificados acima, que consideram três níveis hierárquicos de causalidade. As seguintes co-variáveis foram incluídas na análise segundo os diversos níveis de determinação: a) bloco de variáveis sócio-demográficas: idade da mãe (15-19, 20-28, $\geq 29$ anos), renda familiar total em quartis $(<281,0 ; 281,0-478,5$; $478,6-842,9 ; \geq 843,0$ reais), escolaridade (1-4, 5-8, 9-11 anos), cor de pele segundo classificação do entrevistador (branca, parda/negra), estado marital (casada, em união, solteira), trabalho durante a gravidez ( $\operatorname{sim}$, não) e hábito de fumar ( $\operatorname{sim}$, não); b) bloco de variáveis reprodutivas: idade da mãe ao primeiro parto ( $<19,20-28, \geq 29$ anos), paridade (1, $2, \geq 3$ filhos), ligadura tubária ( $\operatorname{sim}$, não), aborto prévio em alguma gravidez anterior (sim, não), tipo de parto da gravidez index (vaginal, cesárea) e; c) bloco de variáveis relacionadas ao estado nutricional materno pré-gestacional: índice de massa corporal (IMC) $<18,5 \mathrm{~kg} / \mathrm{m}^{2}$ (sim, não), IMC $\geq 25 \mathrm{~kg} / \mathrm{m}^{2}$ (sim, não) e IMC $\geq 30 \mathrm{~kg} / \mathrm{m}^{2}$ ( $\operatorname{sim}$, não).

Os dados de peso e estatura foram coletados segundo procedimentos padronizados, 23 pelo pesquisador principal (GK), e foram utilizados para o cálculo do IMC "peso $(\mathrm{kg}) /$ estatura $\left(\mathrm{m}^{2}\right)$." Os pontos de corte adotados para classificação do estado nutricional foram os definidos pela World Health Organization (WHO) 24 : IMC $\geq 25 \mathrm{~kg} / \mathrm{m}^{2}$ para definir sobrepeso, IMC $\geq 30 \mathrm{~kg} / \mathrm{m}^{2}$ para definir obesidade e IMC $<18,5 \mathrm{~kg} / \mathrm{m}^{2}$ para definir baixo peso.

Para a análise dos dados, inicialmente, investigou-se a distribuição de cada co-variável da amostra com objetivo de definir as melhores categorias. Nessa mesma fase da análise, calculou-se a prevalência de cesárea e aborto segundo cada uma das variáveis dos três níveis hierárquicos descritos acima. As prevalências foram comparadas por meio do teste do qui-quadrado para proporções. Na análise bivariada, utilizou-se como medida de efeito o odds ratio (OR) bruto, intervalo de confiança de $95 \%$ (IC95\%) e o teste do qui-quadrado, para verificar as associações entre as variáveis. Em situações onde se observou tendência linear, utilizou-se o quiquadrado de tendência. Valores de $p<0,05$ foram considerados como estatisticamente significantes.

Como os dois desfechos reprodutivos apresentam distribuição binária, o procedimento de análise multivariada escolhido foi o de regressão logística. Adotou-se a estratégia proposta por Victora et al.,25 que utiliza modelos hierarquizados. De acordo com essa estratégia, todas as variáveis com valores de $p<0,20$ na análise bivariada são selecionadas para análise multivariada, com o objetivo de evitar a exclusão de variáveis potencialmente importantes. Utilizou-se a seleção backwards a partir do modelo saturado, ou seja, todas as variáveis do mesmo nível foram incluídas e depois retiradas, uma a uma, do modelo saturado quando o $p$ valor foi $\geq 0,05$. Dessa forma, os odds ratio e valores de $p$ apresentados foram ajustados para as variáveis do mesmo nível hierárquico e para os níveis superiores. A associação foi avaliada com o teste da razão de máxima verossimilhança (likelihood-ratio test) e para avaliar a qualidade do ajuste em cada nível hierárquico e do modelo final de regressão logística, utilizou-se o goodness-of-fit test. Todas as análises foram realizadas no pacote estatístico Stata 8.0.

\section{Resultados}

Não se observou perda seletiva quando foram comparadas as 479 mulheres que ingressaram na coorte e as 352 que alcançaram o terceiro seguimento, em relação à diversas variáveis socioeconômicas e do estado nutricional (resultados não apresentados).

A Tabela 1 apresenta dados sobre a distribuição 
Prevalência de cesárea e Odds Ratio bruto (OR), segundo variáveis selecionadas, em mulheres no pós-parto. Rio de Janeiro, 1999 a 2001.

\begin{tabular}{|c|c|c|c|c|c|}
\hline \multirow[t]{2}{*}{ Variáveis } & \multirow{2}{*}{$\frac{\text { Amostra }}{n}$} & \multirow{2}{*}{$\frac{\text { Cesárias }}{\mathrm{n}}$} & \multirow{2}{*}{$\begin{array}{c}\text { Prevalência } \\
\%\end{array}$} & \multirow[t]{2}{*}{ OR bruto (IC95\%) } & \multirow[t]{2}{*}{ Valor $p$} \\
\hline & & & & & \\
\hline \multicolumn{6}{|l|}{ Faixa etária (anos) } \\
\hline $15-19$ & 75 & 23 & 30,7 & 1,00 & \\
\hline $20-28$ & 180 & 64 & 35,5 & $1,25(0,69-2,22)$ & 0,453 \\
\hline$\geq 29$ & 97 & 41 & 42,2 & $1,65(0,88-3,12)$ & 0,120 \\
\hline \multicolumn{6}{|l|}{ Renda familiar total (reais) } \\
\hline $1^{\circ}-3^{\circ}$ quartil $(<843,0)$ & 264 & 85 & 32,2 & 1,00 & \\
\hline $4^{\circ}$ quartil $(\geq 843,0)$ & 88 & 43 & 48,9 & $2,01(1,20-3,39)$ & 0,004 \\
\hline \multicolumn{6}{|l|}{ Ler carta ou jornal } \\
\hline $\operatorname{Sim}$ & 250 & 98 & 39,2 & $1,55(0,94-2,54)$ & 0,084 \\
\hline Não & 102 & 30 & 29,4 & 1,00 & \\
\hline \multicolumn{6}{|l|}{ Escolaridade (anos)* } \\
\hline $1-8$ & 244 & 80 & 32,8 & 1,00 & \\
\hline $9-11$ & 104 & 46 & 44,2 & $1,62(1,01-2,60)$ & 0,043 \\
\hline \multicolumn{6}{|l|}{ Cor } \\
\hline Branca & 133 & 62 & 46,6 & $2,02(1,29-3,16)$ & 0,002 \\
\hline Parda/Negra & 219 & 66 & 30,1 & 1,00 & \\
\hline \multicolumn{6}{|l|}{ Estado marital } \\
\hline Casada & 67 & 26 & 38,8 & $1,39(0,70-2,75)$ & 0,339 \\
\hline Vive em união & 205 & 77 & 37,5 & $1,32(0,76-2,29)$ & 0,319 \\
\hline Solteira & 80 & 25 & 31,2 & 1,00 & \\
\hline \multicolumn{6}{|l|}{ Trabalho durante gravidez } \\
\hline $\operatorname{Sim}$ & 170 & 72 & 42,3 & $1,65(1,07-2,56)$ & 0,024 \\
\hline Não & 182 & 56 & 30,7 & 1,00 & \\
\hline \multicolumn{6}{|l|}{ Hábito de fumar } \\
\hline Sim & 50 & 13 & 26,0 & 1,00 & \\
\hline Não & 302 & 115 & 38,0 & $1,75(0,89-3,43)$ & 0,103 \\
\hline \multicolumn{6}{|c|}{ Idade ao primeiro parto (anos)* } \\
\hline$\leq 19$ & 181 & 63 & 34,8 & $1,07(0,41-2,78)$ & 0,893 \\
\hline $20-28$ & 150 & 58 & 38,6 & $1,26(0,48-3,31)$ & 0,638 \\
\hline$\geq 29$ & 21 & 7 & 33,3 & 1,00 & \\
\hline \multicolumn{6}{|c|}{ Paridade (número de filhos) } \\
\hline 1 & 159 & 60 & 37,7 & $1,23(0,71-2,13)$ & 0,454 \\
\hline 2 & 105 & 39 & 37,1 & $1,20(0,66-2,18)$ & 0,544 \\
\hline$\geq 3$ & 88 & 29 & 32,9 & 1,00 & \\
\hline \multicolumn{6}{|l|}{ Ligadura } \\
\hline $\operatorname{Sim}$ & 29 & 26 & 89,6 & 1,00 & $<0,001$ \\
\hline Não & 323 & 102 & 31,5 & $18,8(5,55-63,47)$ & \\
\hline \multicolumn{6}{|c|}{ IMC pré-gestacional $\leq 18,5\left(\mathrm{~kg} / \mathrm{m}^{2}\right)$} \\
\hline $\operatorname{sim}$ & 35 & 14 & 40,0 & $1,18(0,57-2,43)$ & 0,656 \\
\hline Não & 263 & 95 & 36,1 & 1,00 & \\
\hline \multicolumn{6}{|c|}{ IMC pré-gestacional $\geq 25,0\left(\mathrm{~kg} / \mathrm{m}^{2}\right)$} \\
\hline $\operatorname{sim}$ & 74 & 30 & 45,9 & $1,25(0,73-2,14)$ & 0,415 \\
\hline Não & 224 & 79 & 33,9 & 1,00 & \\
\hline \multicolumn{6}{|c|}{ IMC pré-gestacional $\geq 30,0\left(\mathrm{~kg} / \mathrm{m}^{2}\right)$} \\
\hline $\operatorname{sim}$ & 14 & 6 & 35,7 & $1,32(0,44-3,90)$ & 0,618 \\
\hline Não & 284 & 103 & 37,0 & 1,00 & \\
\hline Prevalência geral & 352 & 128 & 36,3 & & \\
\hline
\end{tabular}

* Quatro casos de escolaridade igual a zero foram eliminados da análise. Não foram observados casos de escolaridade superior a 11 anos; IMC=índice de massa corporal. 
Prevalência de aborto e Odds Ratio bruto (OR), segundo variáveis selecionadas, em mulheres no pós-parto. Rio de Janeiro, 1999 a 2001.

\begin{tabular}{|c|c|c|c|c|c|}
\hline \multirow[t]{2}{*}{ Variáveis } & Amostra & Abortos & Prevalência & OR bruto (IC95\%) & Valor $p$ \\
\hline & $\mathrm{n}$ & $\mathrm{n}$ & $\%$ & & \\
\hline \multicolumn{6}{|l|}{ Faixa etária (anos) } \\
\hline $15-19$ & 75 & 15 & 20,0 & 1,00 & \\
\hline $20-28$ & 180 & 52 & 28,9 & $1,62(0,85-3,12)$ & 0,144 \\
\hline$\geq 29$ & 97 & 53 & 54,6 & $4,82(2,41-9,63)$ & $<0,001$ \\
\hline \multicolumn{6}{|l|}{ Renda familiar total (reais) } \\
\hline $1^{\circ}$ quartil $(<281,0)$ & 90 & 26 & 28,9 & 1,00 & \\
\hline $2^{\circ}$ quartil $(281,0-478,5)$ & 86 & 28 & 32,6 & $1,19(0,62-2,26)$ & 0,598 \\
\hline $3^{\circ}$ quartil $(478,6-842,9)$ & 88 & 30 & 34,1 & $1,27(0,67-2,40)$ & 0,455 \\
\hline $4^{\circ}$ quartil $(\geq 843,0)$ & 88 & 36 & 41,0 & $1,70(0,91-3,18)$ & 0,094 \\
\hline \multicolumn{6}{|l|}{ Ler carta ou jornal } \\
\hline $\operatorname{sim}$ & 250 & 86 & 34,4 & $1,05(0,64-1,71)$ & 0,848 \\
\hline Não & 102 & 34 & 33,3 & 1,00 & \\
\hline \multicolumn{6}{|l|}{ Escolaridade (anos)* } \\
\hline $1-4$ & 91 & 38 & 41,7 & 1,00 & \\
\hline $5-8$ & 153 & 49 & 32,0 & $1,69(0,93-3,05)$ & 0,083 \\
\hline $9-11$ & 104 & 31 & 29,8 & $1,11(0,65-1,90)$ & 0,706 \\
\hline \multicolumn{6}{|l|}{ Cor } \\
\hline Branca & 133 & 46 & 34,6 & $1,03(0,66-1,63)$ & 0,879 \\
\hline Parda/Negra & 219 & 74 & 33,7 & 1,00 & \\
\hline \multicolumn{6}{|l|}{ Estado marital } \\
\hline Casada & 67 & 12 & 17,9 & 1,00 & \\
\hline Vive em união & 205 & 82 & 40,0 & $3,05(1,54-6,06)$ & 0,001 \\
\hline Solteira & 80 & 26 & 32,5 & $2,21(1,01-4,81)$ & 0,047 \\
\hline \multicolumn{6}{|l|}{ Trabalho durante gravidez } \\
\hline Sim & 170 & 69 & 40,6 & $1,75(1,12-2,74)$ & 0,013 \\
\hline Não & 182 & 51 & 28,0 & 1,00 & \\
\hline \multicolumn{6}{|l|}{ Hábito de fumar } \\
\hline Sim & 50 & 22 & 44,0 & $1,63(0,89-3,00)$ & 0,113 \\
\hline Não & 302 & 98 & 32,4 & 1,00 & \\
\hline \multicolumn{6}{|c|}{ Idade ao primeiro parto (anos) } \\
\hline$\leq 19$ & 181 & 59 & 32,6 & 1,00 & \\
\hline $20-28$ & 150 & 51 & 34,0 & $0,94(0,59-1,48)$ & 0,787 \\
\hline$\geq 29$ & 21 & 10 & 47,6 & $1,76(0,70-4,43)$ & 0,227 \\
\hline \multicolumn{6}{|l|}{ Paridade (número de filhos) } \\
\hline 1 & 159 & 38 & 23,9 & 1,00 & \\
\hline 2 & 105 & 38 & 36,2 & $1,81(1,05-3,10)$ & 0,031 \\
\hline$\geq 3$ & 88 & 44 & 50,0 & $3,18(1,83-5,54)$ & $<0,001$ \\
\hline \multicolumn{6}{|l|}{ Ligadura } \\
\hline Sim & 29 & 16 & 55,2 & $2,59(1,20-5,59)$ & \\
\hline Não & 323 & 104 & 32,2 & 1,0 & \\
\hline \multicolumn{6}{|c|}{$\begin{array}{l}\text { Índice de massa corporal } \\
\text { pré-gestacional } \leq 18,5\left(\mathrm{~kg} / \mathrm{m}^{2}\right)\end{array}$} \\
\hline Sim & 35 & 7 & 20,0 & 1,00 & \\
\hline Não & 263 & 103 & 39,2 & $2,57(1,08-6,11)$ & 0,032 \\
\hline
\end{tabular}


Prevalência de aborto e Odds Ratio bruto (OR), segundo variáveis selecionadas, em mulheres no pós-parto. Rio de Janeiro, 1999 a 2001.

\begin{tabular}{|c|c|c|c|c|c|}
\hline \multirow[t]{2}{*}{ Variáveis } & Amostra & Abortos & Prevalência & OR bruto (IC95\%) & Valor $p$ \\
\hline & $\mathrm{n}$ & $\mathrm{n}$ & $\%$ & & \\
\hline \multicolumn{6}{|c|}{$\begin{array}{l}\text { Índice de massa corporal } \\
\text { pré-gestacional } \geq 25,0\left(\mathrm{~kg} / \mathrm{m}^{2}\right)\end{array}$} \\
\hline Sim & 74 & 34 & 45,9 & $1,65(0,97-2,82)$ & 0,065 \\
\hline Não & 224 & 76 & 33,9 & 1,00 & \\
\hline \multicolumn{6}{|c|}{$\begin{array}{l}\text { Índice de massa corporal } \\
\text { pré-gestacional } \geq 30,0\left(\mathrm{~kg} / \mathrm{m}^{2}\right)\end{array}$} \\
\hline $\operatorname{Sim}$ & 14 & 5 & 35,7 & $0,95(0,31-2,90)$ & 0,924 \\
\hline Não & 284 & 105 & 37,0 & 1,00 & \\
\hline Prevalência geral & 352 & 120 & 34,0 & & \\
\hline
\end{tabular}

* Quatro casos de escolaridade igual a zero foram eliminados da análise. Não foram observados casos de escolaridade superior a 11 anos.

Tabela 3

Modelo final de regressão logística hierarquizada para ocorrência de cesárea e ocorrência de aborto em mulheres no pós-parto. Rio de Janeiro, 1999 a 2001

\begin{tabular}{|c|c|c|c|}
\hline Variáveis & OR ajustado (IC95\%) & Valor $p^{*}$ & Valor $p * *$ \\
\hline & Ocorrência de cesárea & & \\
\hline \multicolumn{4}{|l|}{ Nível 1} \\
\hline Cor de pele & & & 0,019 \\
\hline Parda/negra & 1,00 & & \\
\hline Branca & $2,12(1,33-3,42)$ & 0,002 & \\
\hline \multicolumn{4}{|l|}{ Nível 2} \\
\hline Ligadura tubária & & & $<0,001$ \\
\hline Não & 1,00 & & \\
\hline Sim & $19,68(5,77-67,15)$ & $<0,001$ & \\
\hline \multicolumn{4}{|c|}{ Ocorrência de aborto } \\
\hline \multicolumn{4}{|l|}{ Nível 1} \\
\hline Faixa etária (anos) & & & $<0,001$ \\
\hline $15-19$ & 1,00 & & \\
\hline $20-28$ & $1,67(0,86-3,24)$ & 0,132 & \\
\hline$\geq 29$ & $6,11(2,94-12,72)$ & $<0,001$ & \\
\hline \multicolumn{4}{|l|}{ Estado marital } \\
\hline Casada & 1,00 & & $<0,001$ \\
\hline Vive em união & $4,22(2,03-8,78)$ & $<0,001$ & \\
\hline Solteira & $3,70(1,59-8,61)$ & 0,002 & \\
\hline
\end{tabular}

*Teste de Wald; **Teste da razão de máxima verossimilhança de frequiência, de prevalência e de OR bruto para cesárea segundo variáveis selecionadas. A prevalência de cesárea foi de $36,4 \%$ (IC95\%: 31,341,6). As maiores prevalências de cesárea foram observadas em mulheres que fizeram ligadura $(89,6 \%)$, pertencentes a famílias com o maior quartil de renda $(48,9 \%)$, de cor branca $(46,6 \%)$, com IMC pré-gestacional $\geq 25 \mathrm{~kg} / \mathrm{m}^{2}(45,9 \%)$ e com escolaridade entre 9 e 11 anos $(44,2 \%)$. Observou-se risco estatisticamente significante para ocorrência de cesárea para mulheres pertencentes ao mais alto quartil de renda em comparação com os três quartis inferiores (OR=2,01; IC95\%: 1,20-3,39), com escolaridade entre 9 e 11 anos (OR=1,62; IC95\%: 1,01$2,60)$, cor de pele branca $(\mathrm{OR}=2,02$; IC95\%: 1,29 $3,16)$, que trabalharam durante a gravidez $(\mathrm{OR}=1,65$; IC95\%: $1,07-2,56)$ e que fizeram ligadura $(\mathrm{OR}=18,8$, IC95\%: 5,55-63,47).

A Tabela 2 apresenta dados sobre a distribuição de frequiência, de prevalência e de OR bruto para aborto segundo variáveis selecionadas. A prevalência de aborto foi de 34,1\% (IC95\%: 29,1-39,3). As maiores prevalências de aborto foram observadas em mulheres pertencentes a faixa etária $\geq 29$ anos $(54,6 \%)$, com três ou mais filhos $(50,0 \%)$, que tinham 29 anos ou mais no momento do primeiro parto $(47,6 \%)$, com IMC pré-gestacional $\geq 25,0$ $\mathrm{kg} / \mathrm{m}^{2}(45,9 \%)$ e fumantes $(44,0 \%)$. Observou-se risco estatisticamente significante de aborto para mulheres na faixa etária $\geq 29$ anos (OR=4,82; IC95\%: 2,41-9,63), que vivem em união $(\mathrm{OR}=3,05$; IC95\%: $1,54-6,06)$, solteiras (OR=2,21; IC95\%: 1,01-4,81), que trabalharam durante a gravidez $(\mathrm{OR}=1,75$; 
IC95\%: 1,12-2,74), com dois filhos $(\mathrm{OR}=1,81$; IC95\%: 1,02-3,21), com três ou mais filhos $(\mathrm{OR}=3,18$; IC95\%: 1,76-5,76), que fizeram ligadura $(\mathrm{OR}=2,59$; IC95\%: 1,20-5,59) e com IMC pré-gestacional $\geq 18,5(\mathrm{OR}=2,58$; IC95\%: 1,02-6,74).

Os resultados do modelo final multivariado hierarquizado para os dois desfechos são apresentados na Tabela 3. Observa-se que mulheres brancas e mulheres que tenham praticado ligadura tubária apresentaram 2,02 (IC95\%: 1,29-3,16) e 19,68 (IC95\%: 5,77-67,15) vezes mais chance de realizar cesárea, respectivamente. Em relação a ocorrência de aborto, observa-se que mulheres com 29 anos ou mais, mulheres em união e mulheres solteiras, apresentaram 6,11 (IC95\%: 2,94-12,72), 4,22 (IC95\%: 2,03-8,78) e 3,70 (IC95\%: 1,59-8,61) vezes mais chances de realizar aborto, respectivamente.

\section{Discussão}

Os dois modelos hierárquicos multivariados apresentados no presente estudo revelaram resultados que merecem atenção. No primeiro modelo observou-se que mulheres de cor de pele branca tinham duas vezes mais chance de realizar cesárea em comparação com mulheres negras ou pardas e que para mulheres que haviam praticado ligadura essa chance foi 19 vezes maior. O segundo modelo revelou que mulheres com 29 anos ou mais tinham seis vezes mais chances de praticar aborto e mulheres que viviam em união ou eram solteiras, aproximadamente quatro vezes mais chance. Esses resultados corroboram a importância em se investigar desfechos reprodutivos como a cesárea e o aborto.

De acordo com a literatura, os riscos associados à cesárea incluem maior freqüência de infecção e hemorragias maternas, maiores índices de prematuridade e de síndrome do desconforto respiratório do recém-nascido. ${ }^{2,4}$ Alguns estudos revelaram que a idade materna superior a 34 anos, a primiparidade, a síndrome hipertensiva, a gemelaridade e a ruptura prematura das membranas, bem como a obesidade, o ganho de peso gestacional excessivo e a deficiência de cálcio são os principais fatores de risco para a cesárea.4,7,26-28

Segundo Kabir et al. ${ }^{29}$ a taxa de cesárea repetida é potencialmente maior em mulheres de cor de pele branca, quando comparada às mulheres de pele negra. Linton et al. ${ }^{30}$ observaram que a cesárea ocorreu duas ou mais vezes em mulheres negras, porém, em outro estudo os autores observaram que o aumento da idade materna acarretou elevação do número de cesáreas em mulheres brancas. 31 Esses resultados são semelhantes ao encontrado na presente investigação, o qual demonstra que a chance de realizar cesárea foi maior em mulheres brancas quando comparadas com mulheres pardas ou negras.

Uma interessante discussão pode ser feita sobre a realização de cesárea e ligadura no mesmo parto. É importante frisar que o desenho do presente estudo não permite essas conclusões devido a questões de temporalidade com que os eventos foram observados, entretanto observou-se na análise multivariada uma chance quase 19 vezes maior de cesárea entre mulheres que realizaram concomitantemente ligadura tubárea. Ao longo das últimas décadas inúmeros avanços da medicina transformaram a cesárea em uma alternativa segura ao parto vaginal quando há algum risco para a mulher ou para o feto. Entretanto, desde a década de setenta, as indicações de cesárea vêm crescendo indiscriminadamente, e esse procedimento cirúrgico utilizado de forma abusiva em vários países.4 Dias e Deslandes32 revelam evidências de que as mulheres brasileiras podem estar optando em fazer cesárea em função do interesse em realizar a ligadura na seqüência. De acordo com Faúndes et al.33, há uma clara relação entre realização de cesárea e ligadura no mesmo parto, já que 70 a $80 \%$ das mulheres investigadas por esses autores realizaram ambos procedimentos no mesmo parto.

Embora nenhum outro fator além da cor de pele e da ligadura tenham apresentado associação com cesárea no presente estudo, algumas investigações recentes como D'Orsi et al. 8 revelaram o efeito de outros fatores no maior risco de cesárea como a primiparidade $(\mathrm{OR}=3,80)$, mulheres cujo parto foi realizado por obstetra do serviço privado $(\mathrm{OR}=1,5)$, e em mulheres que haviam realizado cesárea prévia $(\mathrm{OR}=15,5)$. Da mesma forma, Freitas et al. 34 observaram em um estudo sobre a desigualdade social na distribuição dos riscos de cesárea, que a razão de chance (RC) foi maior para mulheres com baixa escolaridade, de idade materna mais elevada e com maior número de consultas de pré-natal $(\mathrm{RC}=2,16)$.

A freqüência de cesárea no presente estudo foi de $36,4 \%$. Esse valor é muito próximo ao valor de $37,4 \%$ observado por Seligman et al., ${ }^{7}$ com base em dados de 4486 gestantes de seis capitais brasileiras, estudadas entre 1991 e 1996. Essas comparações conferem validade externa aos dados aqui apresentados. Por outro lado, é interessante observar que no estudo de Seligman et al. ${ }^{7}$ a obesidade pré-gestacional e o ganho de peso gestacional excessivo foram considerados fatores de risco independentes para a cesárea, mesmo após o controle para fatores 
de confusão como cor de pele, idade, educação e capital do estudo, associações que não foram identificadas no presente estudo.

A relação com a paridade foi significativa apenas no modelo não ajustado, significando maior chance de aborto quanto maior o número de filhos (prevalência de $50 \%$ para $\geq 3$ filhos versus $23,9 \%$ para primíparas). No estudo de Menezes et al.12 foi observado que $16,7 \%$ das mulheres reportaram a realização de aborto induzido na primeira gravidez, enquanto para os homens, em relação a suas parceiras, esse valor foi bem mais alto $(45,9 \%)$. Incluir a percepção masculina sobre a ocorrência do aborto tem sido uma tendência em estudos recentes.

No presente estudo verificou-se uma elevada frequiência de aborto em mulheres solteiras ou que viviam em união. A chance de praticar aborto foi de três a quatro vezes maior para essas mulheres quando comparadas às casadas, com base no modelo multivariado. $\mathrm{O}$ estado marital deve ser considerado variável proxi para o suporte social, o que explica em parte a maior chance de praticar aborto para mulheres não casadas. Fatores como a falta de planejamento da gravidez também têm sido associados ao aumento da incidência de aborto. 35 Os resultados aqui apresentados estão em acordo com o observado por Olinto e Moreira-Filho, 11 em um estudo de base populacional, no qual mulheres em união tiveram chance duas vezes maior de praticar aborto. A associação para mulheres separadas foi mais tênue $(\mathrm{OR}=1,87)$ e não foi observada entre solteiras. Em um outro modelo de análise multivariada desenvolvido pelos mesmos autores, 11 observou-se risco três vezes maior de aborto associado ao estado marital quando essa variável foi substituída pela variável morar com companheiro. No estudo de Menezes et al.,12 a falta de estabilidade na relação marital foi considerada fator de risco para o aborto quando as análises foram controladas para o efeito de variáveis socioeconômicas.

O estado nutricional pré-gestacional medido pelo IMC não esteve associado aos desfechos estudados na presente pesquisa após controle para variáveis de confusão. Entretanto, é importante ressaltar que o IMC pré-gestacional $\geq 25 \mathrm{~kg} / \mathrm{m}^{2}$ esteve associado a uma maior chance de aborto, com significância estatística limite na análise não ajustada, não podendo ser desconsiderada a prevalência de $45,9 \%$ de aborto para mulheres nessa categoria de IMC. Os resultados da análise bivariada também revelaram maior chance de aborto para mulheres com IMC prégestacional $\geq 18,5 \mathrm{~kg} / \mathrm{m}^{2}$, no entanto, todos os resultados perderam efeito no modelo ajustado. Sugere-se que em futuros estudos o estado nutricional seja considerado de forma mais detalhada em análises sobre desfechos reprodutivos.

Entre as limitações do estudo, devemos destacar que a amostra não foi calculada para identificar os fatores associados aos desfechos reprodutivos aqui investigados, apesar de ter sido suficiente para identificar diversos fatores potencialmente associados.

Em síntese, conclui-se que a cor de pele branca e a prática de ligadura foram co-variáveis potencialmente associadas à ocorrência de cesárea, enquanto que o estado marital em união ou solteira e a idade materna apresentaram potencial associação com a ocorrência de aborto. Não se identificou efeito do estado nutricional pré-gestacional em nenhum dos dois desfechos estudados.

\section{Agradecimentos}

Gilberto Kac recebeu bolsa de doutorado CAPES (1999-2002). O projeto de pesquisa original foi financiado pelas seguintes fontes: Fundação Universitária José Bonifácio, da Universidade Federal do Rio de Janeiro (FUJB/UFRJ) e Fundação de Amparo à Pesquisa do Estado do Rio de Janeiro (FAPERJ). Gilberto Kac é Pesquisador do CNPq. 


\section{Referências}

1. Zugaib M, Nomura RMY. Cesárea a pedido. Bol Inform. 2006; jul./set. Disponível em: http://www.sorgirgs.org.br/Boletim/cesarea.html. [2007 fev 2].

2. Cunha AA, Portela MC, Amed AM, Camano L. Modelo preditivo para cesariana com uso de fatores de risco. Rev Bras Ginecol Obstet. 2002; 24: 21-8.

3. Tedesco RP, Maia Filho NL, Mathias L, Benez AL, Castro VCL, Bourroul GM, Reis FI. Fatores determinantes para as expectativas de primagestas acerca da via de parto. Rev Bras Ginecol Obstet. 2004; 26: 791-8.

4. Cabral SAL, Costa CFF, Cabral SF. Correlação entre idade materna, paridade, gemelaridade, síndrome hipertensiva e ruptura prematura de membranas e a indicação de parto cesárea. Rev Bras Ginecol Obstet. 2003; 25: 739-44.

5. Brasil. Ministério da Saúde. DATASUS. Disponível em: http://www.datasus.gov.br/. [2006 set 13].

6. Fabri RH, Silva HSL, Lima RV, Murta EFC. Estudo comparativo das indicações de cesariana entre um hospital público-universitário e um hospital privado. Rev Bras Saúde Matern Infant. 2002; 2: 29-35.

7. Seligman LC, Duncan BB, Branchtein L, Gaio DSM, Mengue SS, Schmidt MI. Obesity and gestational weight gain: cesarean delivery and labor complications. Rev Saúde Pública. 2006; 40: 457-65.

8. D'Orsi E, Chor D, Giffin K, Angulo-Tuesta A, Barbosa GP, Gama AS, Reis AC. Fatores associados à realização de cesáreas em uma maternidade pública do Município do Rio de Janeiro, Brasil. Cad Saúde Pública. 2006; 22: 2067-78.

9. Schor N. Investigação sobre ocorrência de aborto em pacientes de hospital de centro urbano do Estado de São Paulo, Brasil. Rev Saúde Pública. 1990; 24: 144-51.

10. Fonseca W, Misago C, Freitas P, Santos E, Fernandes L, Correia L. Características sócio-demográficas, reprodutivas e médicas de mulheres admitidas por aborto em hospital da região Sul do Brasil. Cad Saúde Pública. 1998; 14: 279-8.

11. Olinto MTA, Moreira-Filho DC. Fatores de risco e preditores para o aborto induzido: estudo de base populacional. Cad Saúde Pública. 2006; 22: 365-75.

12. Menezes GMS, Aquino EML, Silva DO. Induced abortion during youth: social inequalities in the outcome of the first pregnancy. Cad Saúde Pública. 2006; 22: 1431-46.

13. Nakamura MU, Alexandre SM, Santos JFK. Repercussões obstétricas e perinatais do tabagismo (ativo e/ou passivo) na gravidez. São Paulo Med J. 2004; 122: 94-8.

14. Borges G, Tapia-Conyer R, Lopez-Cervantes M. Alcohol consumption and pregnancy in the Mexican national addiction survey. Cad Saúde Pública. 1997; 13: 205-11.

15. Scholl T, Johnson WG. Folic acid: influence on the outcome of pregnancy. Am J Clin Nutr. 2000; 71 (Suppl): 1295S$\mathrm{S} 303$.

16. King J. Determinants of maternal zinc status during pregnancy. Am J Clin Nutr. 2000; 71 (Suppl): 1334S-S43S

17. Sorrentino SR, Lebrão ML. Os abortos no atendimento hospitalar do estado de São Paulo, 1995. Rev Bras Epidemiol. 1998; 1: 256-67.
18. Kac G, Benício MHDA, Velásquez-Meléndez G, Valente JG. Postpartum weight retention among women in Rio de Janeiro: a follow-up study. Cad Saúde Pública. 2003; 19 : 149-61.

19. Kac G, Benício MHDA, Velásquez-Meléndez G, Valente JG, Struchiner CJ. Breastfeeding and postpartum weight retention in a cohort of Brazilian women. Am J Clin Nutr. 2004; 79: 487-93.

20. Kac G, Benício MHDA, Velásquez-Meléndez G, Valente JG, Struchiner CJ. Gestational weight gain and pre-pregnancy weight status influence postpartum weight retention in a cohort of Brazilian women. J Nutr. 2004; 134: 661-6.

21. Kac G, Benício MHDA, Velásquez-Meléndez G, Valente JG. Nine months postpartum weight retention predictors for Brazilian women. Public Health Nutr. 2004; 7: 661-8.

22. CNS (Conselho Nacional de Saúde). Resolução no 196/96 sobre pesquisa envolvendo serem humanos. Bioética. 1996; 4: 415-25.

23. Lohman TG, Roche AF, Martorell R. Anthropometric standardization reference manual. Illinois: Human Kinetics Books; 1988.

24. WHO (World Health Organization). 1995. Physical status: the use and interpretation of anthropometry. Genebra; 1995. (Technical Report Series, 854).

25. Victora CG, Huttly SR, Fuchs SC, Olinto MTA. The role of conceptual frameworks in epidemiological analysis: a hierarchical approach. Int J Epidemiol. 1997; 26: 224-7.

26. Galtier-Dereure F, Boegner C, Bringer J. Obesity and pregnancy: complications and cost. Am J Clin Nutr. 2000; 71 (Suppl): 1242S-8S.

27. Abrams B, Altman SL, Pickett KE. Pregnancy weight gain: still controversial. Am J Clin Nutr. 2000; 71 (Suppl): 1233S-41S

28. Ritchie LD, King JC. Dietary calcium and pregnancyinduced hypertension: is there a relation? Am J Clin Nutr. 2000; 71 (Suppl): 1371S-4S.

29. Kabir AA, Pridijian G, Steinmann WC, Herrera EA, Khan, MM. Racial differences in cesareans: an analysis of U.S. 2001. Obst Gynecol. 2005; 105: 710-8.

30. Linton A, Peterson MR. Effect of preexisting chronic disease on primary cesarean delivery rates by race for births in U.S. military hospitals, 1999-2002. Birth. 2004; 31: 16575.

31. Linton A, Peterson MR, Williams TV. Effect of maternal characteristics on cesarean delivery rates among U.S. Department of Defense healthcare beneficiaries, 19962002. Birth. 2004; 31: 3-11.

32. Dias MA, Deslandes SF. Cesarianas: percepção de risco e sua indicação pelo obstetra em uma maternidade pública no Município do Rio de Janeiro. Cad Saúde Pública. 20: 10916.

33. Faundes A, Costa RG, Padua KS, Perdigão AM. Association of prevalence of tubal ligation and sociodemographic characteristics of women and their partners in the State of São Paulo, Brazil. Cad Saúde Pública. 1998; 14: 49-57. 
34. Freitas PF, Drachler ML, Leite JCC, Grassi PR. Desigualdade social nas taxas de cesariana em primíparas no Rio Grande do Sul. Rev Saúde Pública. 2005; 39: 761-7.

35. Osborn JF, Cattaruzza MS, Spinelli A. Risk of spontaneous abortion in Italy 1978-1995, and the effect of maternal age, gravidity, marital status and education. Am J Epidemiol. 2000; 151: 98-105.

Recebido em 2 de março de 2006

Versão final apresentada em 10 de abril de 2007

Aprovado em 27 de julho de 2007 